\title{
Deux expériences musicales au Musée d'ethnographie de Neuchâtel
}

\section{François Borel}

\section{(2) OpenEdition}

\section{Journals}

Édition électronique

URL : http://journals.openedition.org/ethnomusicologie/585

ISSN : 2235-7688

\section{Éditeur}

ADEM - Ateliers d'ethnomusicologie

Édition imprimée

Date de publication : 1 novembre 2003

Pagination : 137-153

ISBN : 978-2-8257-0863-7

ISSN : $1662-372 X$

\section{Référence électronique}

François Borel, «Deux expériences musicales au Musée d'ethnographie de Neuchâtel », Cahiers

d'ethnomusicologie [En ligne], 16 | 2003, mis en ligne le 16 janvier 2012, consulté le 05 mai 2019. URL : http://journals.openedition.org/ethnomusicologie/585

Ce document a été généré automatiquement le 5 mai 2019.

Tous droits réservés 


\title{
Deux expériences musicales au Musée d'ethnographie de Neuchâtel
}

\author{
François Borel
}

1 Les deux expériences muséographiques musicales dont il est question ici ont eu lieu dans le même musée, mais à deux décennies d'intervalle et dans un contexte passablement différent. Il m'a semblé intéressant d'en présenter parallèlement les intentions de départ, les scénarios respectifs, de les comparer et de faire part de quelques réactions qu'ont suscité ces expositions dans la presse helvétique.

2 Avant 1979, le Musée d'ethnographie de Neuchâtel, alors dirigé par Jean Gabus, privilégiait les expositions "monographiques", centrées sur une ethnie, sa culture matérielle, ses mythes et son univers symbolique - par exemple Les Touaregs (1971), Malgache, qui es-tu? (1973), Les Esquimaux hier, aujourd'hui (1976), etc. - ou sur une thématique "transversale » - À quoi jouent les enfants du monde (1959), Parures et bijoux dans le monde (1961), La main de l'homme (1963), etc. Ce choix était effectué en fonction des missions de recherche que Jean Gabus avait entreprises l'année qui précédait et au cours desquelles il avait constitué des collections d'objets ethnographiques. Dans ces expositions, ce n'est qu'une vitrine, rarement davantage, qui illustrait parfois certains aspects de la culture musicale de la société présentée ou en relation étroite avec le thème. En 1977, il fut décidé de mettre en valeur les collections d'instruments de musique qui avaient récemment fait l'objet d'un inventaire systématique.

3 Or, notre intention n'était justement pas d'aligner des instruments de musique, ni de faire un inventaire des musique non européennes, mais de présenter, par un choix forcément arbitraire, des manifestations musicales étroitement liées aux activités humaines, à la vie, à la religion, en postulant que la notion de musique telle que nous la concevons n'existe pas ailleurs que dans le monde occidental. Autre point fort qui devait ressortir de l'exposition: l'instrument de musique n'a pas d'existence en dehors de sa fonction. Autrement dit, il n'acquiert une signification qu'à travers la fonction qu'il assume au sein de telle ou telle manifestation musicale. Il était donc souhaitable de le présenter en rapport étroit avec son rôle et même, au besoin, ne pas en faire l'objet central du sujet 
choisi, puisqu'il ne représente qu'un élément intermédiaire. Il fallait donc placer l'instrument « en situation » au milieu des autres objets, le mettre en scène dans un cadre évoquant un contexte quotidien ou cérémoniel de la musique.

4 A lui seul, le titre, Musique et sociétés, a posé problème et a donné lieu à de nombreuses discussions avec Jean Gabus, qui n'avait pas forcément la même idée de l'exposition que les deux scénaristes, Ernst Lichtenhahn et l'auteur de ces lignes. Nous souhaitions écrire «musiques » et «sociétés » au pluriel : cette option fut rejetée catégoriquement. Gabus refusait toute incursion dans un relativisme culturel qu'aurait laissé supposer la marque du pluriel aux deux termes. Finalement, il concéda un «s ", mais à « sociétés » seulement.

\section{L'exposition Musique et sociétés (du 12 juin 1977 au 27 mars 1978)}

5 Les principaux arguments devant figurer dans le scénario de l'exposition, même si celle-ci remonte à un quart de siècle, conservent toute leur valeur aujourd'hui.

1. La manifestation musicale est un phénomène culturel de grande importance, lié aux divers moments de la vie (naissance, mariage, mort, rites de passage, etc.), aux activités humaines (travail, artisanat, commerce, repas, etc.), au langage, à la religion, etc.

2. Mais cette manifestation musicale est un phénomène acoustique passager, il se prête mal à l'exposition spatiale. Une exposition traitant de ce sujet doit donc mettre en évidence d'autres aspects de ce phénomène, notamment ses diverses fonctions.

3. Car aujourd'hui, bien que ce soit encore souvent le cas, il ne suffit pas de présenter des instruments de musique. Certes, l'instrument reste l'élément médiateur le plus direct entre l'observateur et le musicien. Mais ses divers aspects organologiques, technologiques et classificatoire, s'ils doivent figurer dans une exposition, sont à présenter à part, afin de ne pas interférer dans l'évocation des fonctions.

4. La musique est le langage du cœur et de l'âme. Pour y rendre sensible le spectateur en contact avec des cultures musicales qui lui sont totalement étrangères, il faut l'aider à dépasser, pour la durée de la visite (et peut-être pour plus longtemps) sa propre culture musicale, en veillant bien à la meilleure compréhension possible. Ce faisant, il faut essayer de ne pas «faciliter » ce rapport, mais plutôt de rendre le visiteur attentif aux différences et l'amener au respect de ces différences.

5. Les divers éléments à présenter (fonctions, mythes, etc.) ne doivent pas faire l'objet d'une énumération, ni d'un classement. Ils doivent être le sujet particulier de l'exposition ou être intégrés et dispersés à l'intérieur de chaque secteur. Il est souhaitable de faire une exposition transparente, sincère, qui évite la généralisation et se limite à certains secteurs précis et connus, sans tendre à une forme d'universalisme qui empêcherait le spectateur de comprendre véritablement les phénomènes. Nous avons donc choisi des sujets précis qui recouvrent un grand nombre des aspects et fonctions envisageables en tenant compte également de ce qui est facilement réalisable et, bien sûr, des limitations de crédit.

6. Dans le souci de laisser au spectateur le choix d'une interprétation différente des phénomènes musicaux qui lui sont présentés, il faut qu'il puisse ressentir, à travers l'exposition, un certain ton de remise en question, d'inachèvement à la lecture des textes qui lui seront soumis. 
Fig. 1 : Juke box à l'entrée de l'exposition Musique et sociétés. Photo MEN, Walter Hugentobler

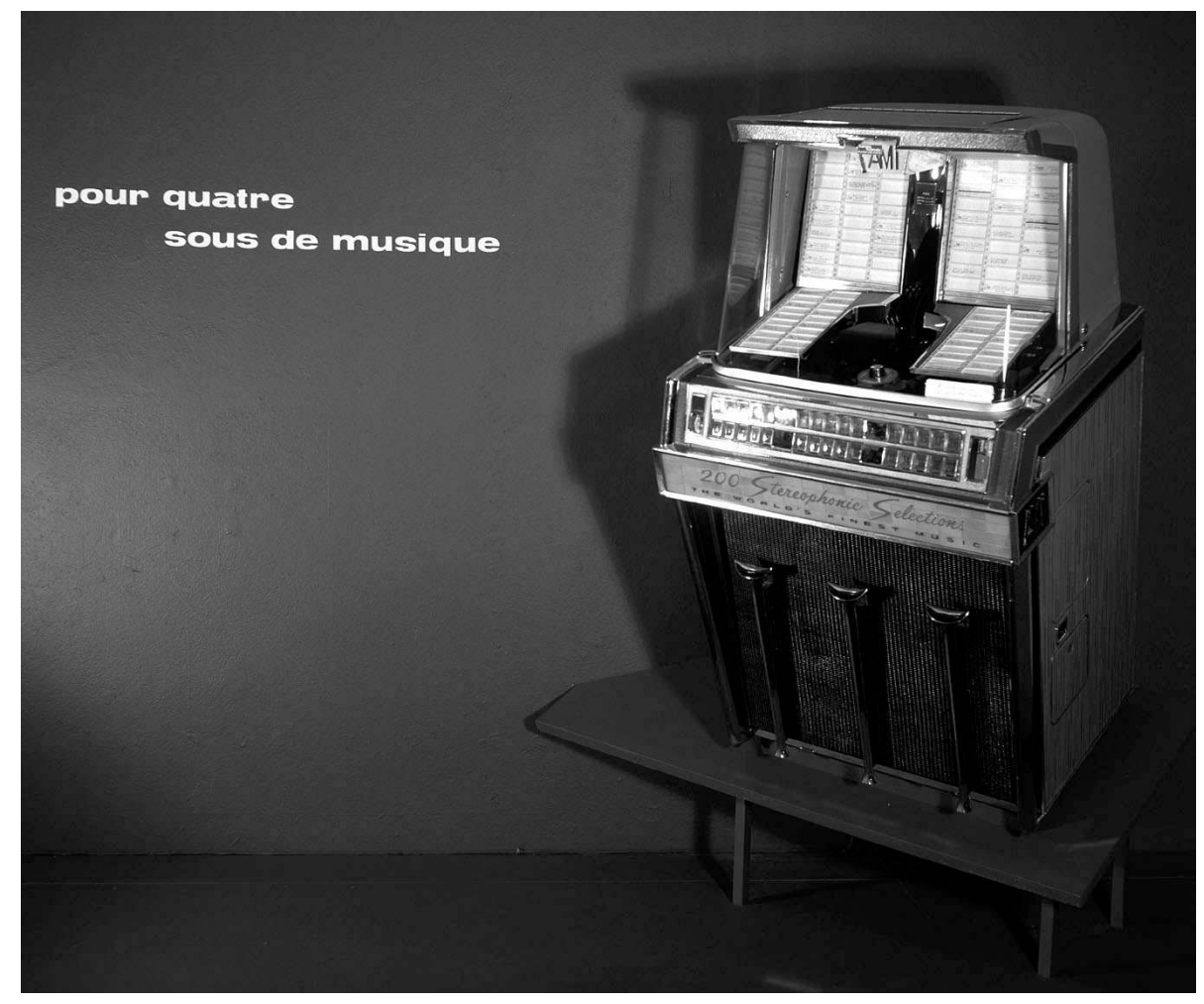

En fonction de ces quelques points forts, le scénario suivant (résumé) fut soumis à la direction, qui l'approuva.

7 Pour quatre sous de musique. À l'entrée, un juke-box visait à faire prendre conscience au visiteur combien la musique de consommation se trouve éloignée de musiques pleines de sens et de fonctions. Suivaient une dizaine de secteurs dans lesquels les instruments de musique étaient mis en scène au milieu d'éléments de reconstitution de l'habitat original et, dans certains cas, renforcés par une illustration sonore ou un montage audiovisuel (dias et son).

8 Les sept notes-couleurs de l'univers de la musique indienne constituaient une sorte de pont avec notre conception de la musique, puisque ces notes laissent deviner une théorie musicale sous-jacente. Instruments présentés : deux vînâ, l'une du Nord, l'autre du Sud de l'Inde ; une paire de tabla et bayan. Autres objets : six figures de temple en bois sculpté représentant des personnages féminins jouant des instruments.

Le baptême de la pirogue chez les Iatmul de Papouasie Nouvelle-Guinée: ici, le son des instruments fait entendre la voix des ancêtres et le créateur originel, le crocodile, se manifeste par le tambour d'eau. Ce secteur, dans lequel était reconstitué le fronton d'une maison commune, était complété par un montage audiovisuel (dias et son). Les instruments exposés étaient au nombre d'une cinquantaine. On y reconnaissait entre autres deux tambours à fente en forme de crocodile, respectivement de 3 et $4 \mathrm{~m}$ de long; de très nombreuses flûtes et trompes traversières décorées; des tambours d'eau; des tambours en sablier; des cithares et arcs musicaux ; de même que les autres éléments du rituel, notamment les accessoires nécessaires aux parures cérémonielles. Tous ces objets, exposés dans des vitrines, avaient été empruntés au Museum für Völkerkunde de Bâle. 

l'agrandissement d'une page de la bande dessinée Oum Pah Pah le peau-rouge confronté au langage tambouriné. Instruments exposés : trois tambours à fente du Cameroun et du Zaïre ; deux tambours d'aisselle du Nigéria et de Côte-d'Ivoire.

11 Les tambours qui mangent.Chaque année à Salvador de Bahia, au cours d'une cérémonie, on nourrit les tambours du candomblé pour entretenir leur force d'invocation des divinités orisha. Instruments exposés: trois tambours capelongo d'Angola ornés de parures rituelles.

12 Le «Bumba-meu-boi».Un exemple de syncrétisme musical: musique, rythme, chorégraphie, thème et costumes dénotent une origine complexe révélant trois composantes de la formation de la culture brésilienne : l'apport amérindien, l'apport africain et l'apport occidental. Le spectacle du «Bumba-meu-boi » constitue l'un des nombreux exemples de syncrétisme musical dans le Nord-Est du Brésil. Son thème met en scène la condition et les rapports de l'esclave vis-à-vis de son maitre, grand propriétaire latifundiaire. Vingt-deux personnages et animaux grandeur nature étaient mis en scène sur une estrade.

La vraie musique est celle de la forêt. Chez les Yukuna d'Amazonie colombienne, les instruments ne sont joués qu'à l'intérieur de la maison des hommes, pour signifier à l'extérieur ce que font les humains à l'intérieur. Les seuls instruments joués à l'extérieur sont les flûtes du Yurupari, dont le son se mêle au fond sonore et grave de la nature. Paradoxalement, ces flûtes étaient absentes du secteur, puisque trop sacrées pour figurer dans les collections. En revanche, les tambours rituels à fente suspendus obliquement, ainsi que les hochets, sonnailles, flûtes de Pan et trompes droites figuraient dans l'esquisse de maison commune matérialisée par un pan de toit en feuilles de palmier. 
Fig. 2 : Le tambour sur mortier touareg tendey

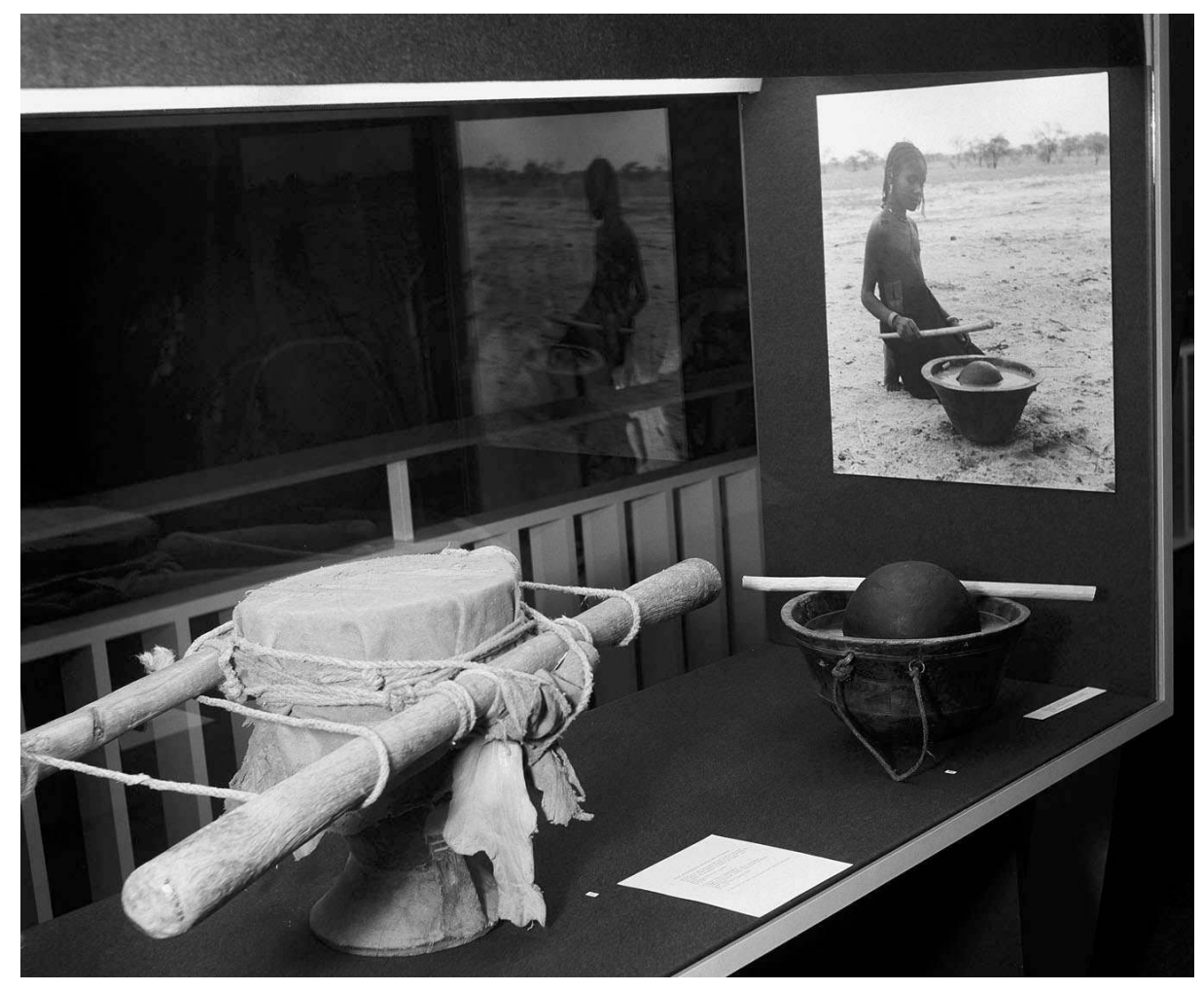

Photo MEN, Walter Hugentobler

De la calebasse aux chants de guérison :la vièle touarègue anzad est parfois utilisée dans le cadre de musiques curatives. En outre, la vièle godié des Zarma ou gogué des Haoussa du Niger est jouée pour favoriser la transe de possession par la divinité qu'on désire consulter. Instruments : vièle monocorde touarègue et djerma.

Le tendé touareg, reflet de la hiérarchie. Le tambour sur mortier est utilisé exclusivement par les femmes de la classe des serviteurs, puisque ce sont elles qui pilent le mil du matin au soir. Comme celui de la vièle monocorde touarègue, le répertoire des chants de tendé comprend de nombreux airs et rythmes de guérison. Pourtant, imzad et tendé ne sont jamais associés. Est-ce le reflet de l'ordre social, fortement hiérarchisé, chez les Touaregs? Ce secteur était composé de vitrines et d'une tente touarègue complète, pourvue de tous ses accessoires, d'une vièle monocorde, d'un tambour sur mortier et d'un tambour d'eau. Dans le même espace, un montage dias-son animait la section.

Instruments de musique haoussa. Les musiciens savent profiter des biens et facilités que leur offre la vie sédentaire, comme des «bienfaits " matériels de la civilisation occidentale : boîtes de conserve, stylo bille, plastique, etc. De même, il est beaucoup plus facile de confectionner une flûte à partir d'un tuyau de plastique qu'au moyen d'une fragile racine d'acacia. Instruments exposés: flûte en matière plastique, deux hautbois et deux tambours.

17 Vitrine «tambourins». A ln-Gall (Niger), les femmes songhai accompagnent leurs chants et danses du rythme du tambourin. Pour en augmenter la force et lui conférer un pouvoir magique, elles le décorent ou le font décorer de formules et de dessins magico-religieux. Instruments exposés : cinq tambours sur cadre, ainsi qu'un recueil de formules magicoreligieuses. 
Certains des secteurs étaient accompagnés d'une illustration sonore, ce qui conférait à l'ensemble une sorte d'atmosphère de foire plutôt agréable :

Les sept notes-couleurs de l'univers (durée: 9'15)

1. Extrait du râga Malkauns : « Ô Seigneur Shiva, Shiva, tu es le maître de la joie... », chanté le soir. Chant, tanpura (luth à 4 cordes) : Mohan LaI Bharai. Enregistrement Laurent Aubert, Népal, 1973, 5'.

2. Extrait du râga Patamanjari. Joué tôt le matin, au printemps. Sitar (luth à $6+12$ cordes) : Mahmud Mirza ; tabla : Wishanath Misra. Enregistré à Bombay, janvier 1977, 4'15.

Les tambours-qui-parlent (durée : 5'25)

1. Tambour à fente, Topoke, Zaïre.

2. Tambour à fente (petit), Bambala, Zaïre.

3. Tambour à membrane ngoma, Bambala, Zaïre.

4. idem, seul.

5. Tambour-d'aisselle, Yorouba, Nigéria.

Les tambours-qui-mangent et Bumba meu boï (durée : 9'15)

1. Tambours à membranes (rum, rumpi, lé), Nord-Est du Brésil, cérémonie pour la divinité Eshu.

2. Tambours et chant, Nord-Est du Brésil, cérémonie Umbanda.

3. Sambinba do Para, Nord-Est du Brésil.

4. Ecole de samba « Mangueira », Rio. Enreg. F. Borel, Rio, 1969.

5. Pedro Pedreiro, chanté par Nara Leão.

La vraie musique est celle de la forêt (durée : 9'30)

Enregistrements effectués en Amazonie colombienne par P.-Y. Jacopin chez les Indiens Yukuna, en 1969-1971. Archives sonores du MEN, Ja 111, 117-120, 123, 127.

1. Trompes sacrées du Yurupari (non exposées, car trop sacrées).

2. Flûte à coulisse, hochets métalliques (boîtes de conserve) et chant (+ trompes sacrées).

3. Tambours à fente, hochets en matière végétale, chant, sifflet ocarina.

4. Trompes droites en balsa décoré.

5. Flûtes de Pan.

Deux autres petites salles de l'exposition étaient consacrées à la systématique des instruments de musique selon Hornbostel et Sachs et divisées en quatre grandes vitrines illustrant les quatre catégories organologiques à travers une centaine d'instruments.

Le bilan de cette exposition peut être résumé de la façon suivante :

- Réaliser une exposition de ce genre comportait un risque d'autant plus grand qu'il n'existait pratiquement pas de modèle (peu d'expériences dans le domaine des expositions " ethnomusicologiques »); il fut d'emblée décidé de ne pas réduire cette exposition à un étalage d'instruments ; l'accès en serait difficile au " grand public ».

- L'itinéraire de l'exposition fut élaboré au Musée de manière à ce que le visiteur remette en question dès le début son propre « contexte » musical, et ceci grâce à une certaine forme de provocation. Les sujets choisis (dont la disparité fut plus ou moins imposée par les circonstances) furent placés dans un certain ordre qui facilitait la transition pour le spectateur : la musique indienne est relativement connue ; elle possède une théorie reposant sur une intellectualisation dans une certaine mesure comparable à la nôtre. De plus, la beauté des objets (instruments) est immédiatement perceptible. Par contre, cette musique est partie intégrante de la vie, de la nature et des croyances. 
- Avec les latmul, l'instrument reste un « bel objet » (art latmul de la sculpture). Mais la différence et la distance prédominent. Le langage tambouriné est l'un des grands mystères des musiques non européennes, et surtout africaines. La musique brésilienne est un exemple, unique en son genre, de syncrétisme musical. L'expression musicale des Yukuna est certainement celle qui reste la plus éloignée de la nôtre. Les instruments utilisés au Sahel reflètent clairement le mode de vie et la structure sociale de ses habitants. Enfin, la systématique des instruments de musique revêt un aspect purement didactique et esthétisant. Elle ne doit pas être considérée comme une fin en soi.

\section{Les échos}

21 Etant donné le risque évoqué plus haut, il était intéressant de connaître les réactions du public à travers l'œil de la critique. Nous étions-nous exprimés assez clairement? Subsistait-il des malentendus ? En guise de réponse, voici quelques extraits de presse. «Dès l'entrée, écrit J. S. dans le Journal de Genève du 20 novembre 1977, un juke-box rappelle, de manière un peu provocante, la musique industrielle $\mathrm{du} \mathrm{XX}^{\mathrm{e}}$ siècle. » Mais sa conclusion est assez conforme à nos intentions: «[...] on constate que la musique occidentale est un acte esthétique individuel, une activité artistique séparée des autres activités autant dans l'espace que dans le temps. La notion de musique elle-même est un concept dont les limites sont beaucoup plus floues dès qu'on s'éloigne de l'Occident. Partie intégrante de fêtes rituelles, étroitement liée à des états seconds, [...] la musique est expression de l'ordre cosmique [...] et associée à la crainte, au sacré, au mystère. » En revanche, Construire (Migros-Zurich, 20 juillet 1977) s'insurge contre ce genre d'approche : «Est-ce que cette entrée en matière, théâtrale à l'excès, se justifie par un besoin impérieux de rompre l'ethnocentrisme dont tout visiteur est immanquablement affligé du seul fait de son appartenance à la race blanche [...] ? Les vues scientifiques semblent en l'occurrence s'être laissé gauchir par des jugements de valeur dont les poncifs de défaitisme intellectuel l'emportent sur la clairvoyance réfléchie. [...] Après tout, il se peut que les ethnomusicologues occidentaux ne connaissent dans leurs sociétés que la musique des juke-box ».

Sous le titre «Davantage de questions que de réponses », le Bund (Berne, $1^{\mathrm{er}}$ septembre 1977) se demande si l'on peut « exposer la musique, présenter des organismes et fonctions sociales à l'intérieur de vitrines, et démontrer les rapports multiples entre musique et société dans les locaux d'un musée » et constate : «Ce ne sont pas là les seules questions qui se posent au visiteur [...]. Les réponses, les explications globales, il ne les trouvera finalement qu'esquissées. »

La Neue Zürcher Zeitung (Zurich, 2 septembre 1977) déclare : « Elle est révolue, l'époque où l'on croyait pouvoir rentrer chez soi avec un chant esquimau enregistré, le disséquer et le 'comprendre' par des méthodes occidentales. [...] Celui qui veut saisir l'essence d'une pratique musicale vieille de plusieurs générations, sinon de plusieurs siècles, doit commencer par envisager cette expression musicale dans le cadre de vie des cultures envisagées. "

Et la Revue musicale suisse (Lausanne, décembre 1977) de conclure : « [...] On ne s'ennuie à aucun moment et on quitte le musée plein d'idées et sans aucun sentiment de fatigue. » 


\section{L'exposition Pom pom pom pom : une invitation à voir la musique (du 31 mai 1997 au 18 janvier 1998)}

En 1997, la seconde exposition s'est préparée dans un tout autre contexte de remise en question des principes muséographiques conventionnels et s'est inscrite dans un cycle de trois expositions successives consacrées à la perception de l'art dans nos sociétés occidentales : successivement Pom pom pom pom (1997), Derrière les images (1998) et L'art c'est l'art (1999).

28 La problématique était ici de présenter une réflexion sur la "bande-son » de notre époque et les activités qui s'y rapportent. Libre d'associer à sa guise les textes, les objets, les sons et les images qui lui étaient présentés, le visiteur était amené à s'interroger sur la manière dont la musique intervient pour influencer sa vision du monde. Pour cela, chaque propos, chaque objet, chaque extrait musical était confronté à un contrepoint engendrant la réflexion (les idées naissent des oppositions).

29 À l'occasion de cette exposition, le MEN a entièrement sonorisé l'espace disponible, ce qui constituait une première dans ces lieux. Après avoir résolument écarté l'usage du casque - histoire d'éviter une « zombification » des visiteurs - et renoncé à l'idée de « douches sonores", trop contraignantes pour la scénographie, nous avions décidé d'assumer pleinement la présence de la musique dans l'espace, en jouant la carte de la «sculpture sonore ». Pour ce faire, un faux plafond fait d'épais panneaux de laine de verre fut installé dans la grande salle. Afin d'éviter la cacophonie, il s'agissait ensuite d'articuler les neuf points sonores de l'exposition. Les commissaires de l'exposition, Marc-Olivier Gonseth et l'auteur de ces lignes, ont développé le concept musical en associant un grand nombre d'extraits musicaux au contenu des secteurs. Le concept a ensuite été discuté avec un musicien genevois, Dominique Barthassat, qui s'est chargé de le développer et de l'enrichir en ajoutant un fond sonore fait de bruits et de sons naturels et synthétiques, ainsi que d'extraits de musique contemporaine. Synchronisés par trois, les neuf points sonores ainsi obtenus diffusaient une composition musicale à la fois globale et focalisée à travers laquelle les visiteurs étaient amenés à se frayer un chemin.

Une sélection d'instruments de musique, n'étant pas "partie prenante » de l'exposition, étaient exposés en hauteur, formant une sorte de frise sur les murs de la grande salle, et « regardaient » en quelque sorte les visiteurs. Ils avaient été choisis en fonction de leurs qualités esthétiques et de leur originalité formelle. 
Fig. 3: Frise d'instruments «spectateurs». Photo MEN, Alain Germond

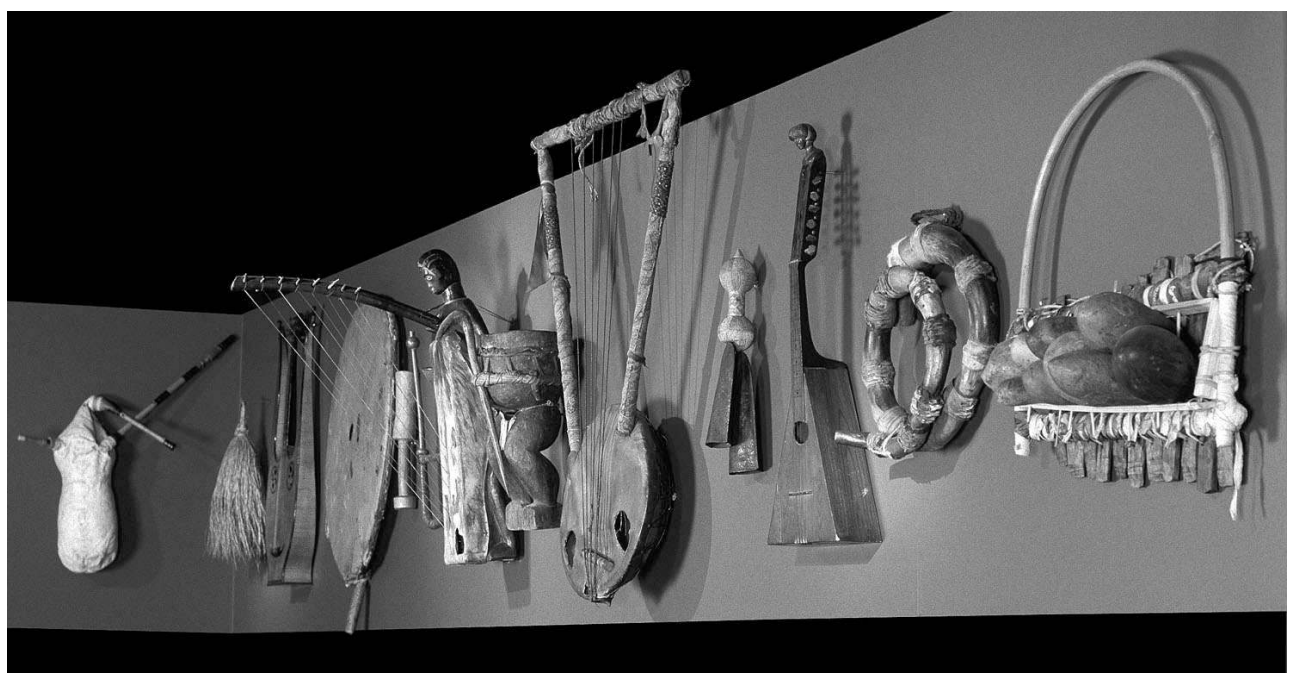

\section{Première partie}

Dans trois espaces fermés successifs, sortes de huttes au caractère intimiste, l'exposition investissait tout d'abord l'espace $d u$ «je», l'expérience intérieure et la dimension cérémonielle : bruissement du silence à partir duquel toute musique s'organise, besoin de faire le vide, offert par la pratique de la méditation ; désir de faire le plein, manifesté par l'attrait des liturgies laïques et religieuses.

1. Le seuil du silence

- Contenu visuel : froid, cellule bleue.

- Contenu musical : le silence ; motif principal : souffle, microcosme sonore.

- Contrepoint : eau, bruits métalliques.

- Tableau d'instruments au mur extérieur : violon muet et cithares sans cordes, Tanzanie.

- Vitrines : œuvre monochrome, partition de John Cage, statuette mende, parfum « Silences » de Giacomo, bague de silence (sorte de labret rituel) senoufo de Côte-d'Ivoire, masque pende du Zaïre à bouche obturée, sourdine de trompette, boules Quiès, marteau de tribunal, sonnette de président de séance, etc.

2. L'appel du vide (la méditation)

- Contenu visuel : lieu de prière.

- Contenu musical : la mélopée ; motif principal : musique tibétaine.

- Contrepoint : chœurs bunun, musique occidentale de méditation.

- Tableau d'instruments au mur extérieur : cloche d'autel bouddhique, tambour à boules fouettantes, trompes.

- Vitrines : œuvre monochrome, feuillets de notation de trompe dung-chen tibétaine, masque bikom du Cameroun, bouddha en méditation du Japon, chapelet musulman, appareil à faire le vide, Lie Tseu : Le vrai classique du vide parfait.

3. L'essence du pein (la transcendance)

- Contenu visuel : autel dans un salon BCBG.

- Contenu musical : musique religieuse intimiste ; motif principal : chant grégorien, aria/ cantate de Bach (Erbarme dich et Ich habe genug).

- Contrepoint : chamanisme yakoute et appel à la prière islamique (muezzin).

- Tableau d'instruments au mur extérieur : tuyaux d'orgue. 
- Vitrines : œuvre monochrome, antiphonaire de chant grégorien (plain-chant) du XIII - XIV $^{\mathrm{e}}$ s. ; figure moba du Togo, livre de messe, maquette d'échelle, de fusée Ariane, panneau de commande d'ascenseur, bouteille de Romanée-Conti 1944.

\section{Deuxième partie}

Dans sa deuxième partie, l'exposition évoquait l'espace du «nous », des rencontres et des affirmations sociales: fusion communautaire à travers le chant, la danse, la fête ; distinction sociale par le recours à un style musical particulier ou par le rejet du goût de l'autre; expression d'une origine ethnique ou nationale par l'emblématique et la typification.

\section{Transes en danses (la fusion communautaire)}

- Contenu visuel : pavillon festif, carnavalesque, sur le modèle du clando africain.

- Contenu musical : la transe ; motif principal : gospel, techno, salsa.

- Contrepoint : musique de guérison, vaudou.

- Vitrines : masques de Venise, tschokwe, masques du Lötschental ; frise décorative de masques africains, indiens et masques de Michael Jackson, de Johnny Hallyday, etc.

\section{Codes en vogue (la distinction sociale)}

- Contenu visuel : un garage, partie extérieure avec pompe à essence-vitrine et vitrine du garage, et partie intérieure avec mobilier contemporain pour présenter des objets attribués clairement à des styles de vie différents.

- Contenu musical : le style ; motif principal : opéra, hard rock, musette, jazz.

- Contrepoint : acousmatique, Satie, chamber music.

- Objets et dérives : objets musicaux, kitsch musical désignant des groupes.

- Vitrines : objets-gadgets liés au look rock, à l'élite jazz, à la distinction classique, au « bal popu » musette.

3. Sens etnik (faire reconnaître)

- Contenu visuel : une boutique ethnique, un bazar.

- Contenu musical : l'emblème régional ou national ; motif principal : tango, musique arabe, reggae, baoulé.

- Contrepoint : flamenco, Ranz des vaches, celtique, Aux armes, etc.

- Objets et dérives : vêtements, nourriture, objets artisanaux et art d'aéroport d'Europe du Sud, du Nord, d'Afrique, des Amériques et du Moyen-Orient.

\section{Troisième partie}

33 Elle abordait l'espace du « ils », l'importance de la musique dans les rapports de force qui structurent les sociétés contemporaines : effets de foule au concert en plein air comme au stade de football ou lors des manifestations de rue; production et consommation de masse au supermarché, à l'aéroport et au restaurant ; alignement et conformité sur les lieux d'endoctrinement idéologique produisant résistance et contestation sur d'autre fronts.

Cadre visuel général: prison transparente, village planétaire dirigé par Big Brother, mélange de fascisme et de convivialité, de contrôle et de révolte sociale. Tapis roulant au centre ; lumière froide ; instruments dans des poubelles (matière première dont on peut se passer, réduits à l'état de déchets, sujets à l'obsolescence, manipulables à souhait, ne ralliant plus par eux-mêmes) : cordophones, idiophones... tambours, guitares... grandes photos sur les murs. 
Objets : signes de pouvoir et signes de révolte.

1. Alignez, couvrez (pouvoir : la mise au pas, la concentration ; contre-pouvoir : la manif)

- Contenu visuel : effet de foule, effet d'alignement.

- Contenu musical : la musique de foule; motif principal : chant de Marines, la musique militaire (Général Guisan), le match de football (Pink Floyd), le Band Aid.

- Contrepoint : le festival rock (Woodstock : no rain), la street parade (Arte), la manifestation (Chicago).

- Objets et dérives : iconographie murale sur toile, foule, manifestation sportive ou politique. Sifflets, fanions, briquets, packs de bière.

2. Liquidation totale (la consommation)

- Contenu visuel : rayon ménager.

- Contenu musical : la musique qui se vend et qui fait vendre; motif principal : la musique de supermarché, de gare, d'aéroport (easy listening, bruits de grands magasins, promotions gigot d'agneau), la variété (boys bands), la soupe fusionnée (world music).

- Contrepoint : la musique invendable, la musique minimaliste.

- Objets et dérives : soupières, paquets de soupe.

3. Tous en chour (l'idéologie)

- Contenu visuel : alignement de bottes de combat.

- Contenu musical : le pouvoir et la contestation, discours totalitaires et libertaires.

- Motif principal : musique de Wagner, Strauss, discours d'Hitler, de Le Pen, Also sprach Zarathustra.

- Contrepoint : transgressions rap (NTM), Hendrix (hymne US).

\section{Epilogue}

L'exposition présentait enfin les nouveaux modes de communication audiovisuels, à travers la projection obsédante d'un tag sonore extrait d'Internet désignant de façon à la fois dérisoire et parodique la puissance et la froideur potentielles des technologies numériques.

Pom pom pom pom constituait une exposition «à la manière du MEN », à la fois caustique, sonore, colorée, esthétique et critique, soulignant la richesse du miroir que nous tendent les formes musicales les plus diverses et interrogeant à travers elles les pratiques, les croyances et les représentations de notre temps.

L'exposition a suscité de nombreux commentaires, dont voici quelques extraits :

«La musique c'est... la musique. Et « Pom pom pom pom », c'est Beethoven. Les gens du Musée d'ethnographie de Neuchâtel ont voulu aller plus loin que ces raccourcis, pour scruter les dessous de notre bain musical quotidien. " (Le Matin, Lausanne, $1^{\mathrm{er}}$ juin 1997)

« En neuf espaces sonores et visuels d'exposition, la musique n'apparaît plus comme innocente. Elle est manipulatrice et exploitée. Inspiratrice ou oppressante. Elle devient un objet de pouvoir, utilisée comme auxiliaire obéissante de l'endoctrinement idéologique ou économique. On la découvre aussi comme instrument propice à la méditation. Elle est le moyen d'influencer les réflexes identitaires. Elle se décline en vêtements et en objets de consommation. Elle s'impose également comme l'expression d'une origine ethnique et participe parfois au rejet de l'autre ou, au contraire, rassemble. » (Coopération, Bâle, 18 juin 1997) «'Pom pom pom pom'. Malgré un titre percutant, croquant Beethoven et les vigoureuses initiales de sa $5^{\text {e }}$ Symphonie, l'exposition du Musée d'ethnographie de Neuchâtel ne cherche pas à mettre en image la musique telle qu'elle est - ou a été 
- pensée, conçue, composée. Elle donne en revanche à voir la musique telle qu'elle est utilisée, perçue, consommée dans les diverses sociétés humaines. Fidèle à sa démarche 'ethnographique', et privilégiant l'effet de surprise, elle fonctionne essentiellement sur la base de la confrontation, volontiers abrupte, entre les symboles de diverses cultures et diverses époques. Le masque de Michael Jackson côtoie ceux de sorciers africains ou des masques de carnaval, le buste de Beethoven se retrouve avec des lunettes en plastique sur le nez. » (L'Hebdo, Lausanne, 3 juillet 1997)

"L'ensemble est terriblement efficace, bouscule avec humour les idées reçues et pousse le visiteur à réfléchir davantage sur l'apparente évidence de certaines valeurs musicales. En sortant de l'exposition, il écoutera à coup sûr d'une oreille différente ses musiques préférées. » (Femina, Lausanne, 6 juillet 1997)

«On vous parlera moins de notes que de résonance. Car la musique a une histoire sociale. Elle a - et elle est - un contexte, une ambiance. Il fallait oser aborder son statut social, le sien et celui de son vilain frangin, le bruit. Les auteurs ne font finalement pas beaucoup de différence entre musique et autres sons. Ils ont un seul contraire : le silence. Mais jamais l'exposition ne s'enlise dans la cacophonie. [...] Avec " pom pom pom pom », encore une fois, le Musée d'ethnographie nous donne des leçons de scénographie. L'expo est un délice de promenade et un grand moment de réflexion. » (Le Nouveau Quotidien, Lausanne, 8 août 1997)

«L'exposition compte sur l'imagination du public. Beaucoup d'objets, de textes d'accompagnement, de poèmes et de commentaires ne dévoilent leur rapport à la musique qu'une fois assimilés dans la tête des spectateurs. Pas d'explications d'ordre didactique, mais beaucoup de simples allusions, et souvent on reste perplexe, à se demander si les exposants se sont facilité la tâche ou si leur imagination fait défaut. [...] Ce qui reste, c'est une impression mitigée, d'autant plus que l'affiche remarquable promet plus que ce que finalement l'exposition arrive à tenir. » (Der Bund, Berne, 19 août 1997)

\section{Conclusion} réalité qui devaient expliquer au visiteur ce qu'était la musique des autres et dans quel contexte elle était censée être produite. L'imagination du visiteur était mise à contribution pour compléter un tant soit peu les lacunes laissées par une scénographie plutôt sommaire et parfois maladroite.

40 paradoxalement peu de place à l'imaginaire en imposant de manière presque autoritaire une réflexion guidée par le tissage serré que constituait la succession d'objets, de textes, de poèmes et de sons.

41 Si Musique et sociétés s'inscrivait dans une muséographie traditionnelle "grand public », faisant la part belle à une lisibilité immédiate, «terme à terme », elle s'inspirait aussi d'une ethnomusicologie privilégiant l'approche anthropologique de la musique et l'organologie. Avec Pom pom pom pom, ces principes ont été remis en question et ont fait appel à une vision sociétale au deuxième degré du rôle de la musique, en incitant le visiteur à se poser des questions plutôt qu'à lui servir des réponses. Ce faisant, les auteurs de l'exposition ont aussi souhaité attribuer un nouveau rôle à l'ethnomusicologie, celui d'envisager l'étude des représentations de la musique dans nos sociétés occidentales contemporaines et plus seulement celui de faire étalage de connaissances qu'elle prête à des groupes sociaux mais dont, finalement, elle ne peut garantir l'authenticité. 
rait alors possible de résoudre ce problème en réalisant une exposition qui fasse étalage des représentations que nous nous faisons de la musique d'une société donnée, comment nous l'entendons, comment elle est reliée à nos schémas de perception à travers notre culture musicale. Parallèlement, il serait intéressant de montrer le travail de l'ethnomusicologue en expliquant le processus d'enquête dans son ensemble, avec ses incertitudes, ses questions, ses doutes, les problèmes liés à l'acculturation, au changement, aux surprises que réservent ces changements par rapport aux idées reçues, aux clichés et raccourcis que notre société utilise couramment. Elle devrait aussi être complétée d'un point de vue symétrique, celui des membres de la société étudiée quant à notre propre musique occidentale, qu'elle soit "classique » ou de variétés. Peut-être arriverait-on ainsi à présenter au public une sorte de phénomène social total qui expliquerait mieux la place de la musique dans une société.

\section{BIBLIOGRAPHIE}

BOREL François et Ernst LICHTENHAHN, 1978, «L'exposition 'Musique et sociétés'», in : Ville de Neuchâtel. Bibliothèques et Musées 1977 : 125-127.

BOREL François, Marc-Olivier GONSETH, Jacques HAINARD et Roland KAEHR éds, 1997, Pom pom pom pom : musiques et caetera (catalogue d'exposition). Neuchâtel : Musée d'ethnographie.

BOREL François, Marc-Olivier GONSETH, Jacques HAINARD et Roland KAEHR, 1998, « Musée d'ethnographie », Bibliothèques et Musées de la Ville de Neuchâtel 97 (Neuchâtel) : 137-168.

GHK, 1997, Pom pom pom pom : une invitation à voir la musique (Texpo 4). Neuchâtel : Musée d'ethnographie.

LICHTENHAHN Ernst, 1977, « Exposer la musique ? », in: Musique et sociétés (catalogue de l'exposition). Neuchâtel : Musée d'ethnographie : 41-44.

\section{RÉSUMÉS}

Deux expositions, réalisées à vingt ans d'intervalle au MEN, ont eu pour thème la musique. La comparaison des approches thématiques, des scénarios et des réalisations scénographiques de chacune d'entre elles, ainsi que des commentaires critiques parus dans la presse permet de se faire une idée de l'évolution de la manière de présenter la musique dans un musée d'ethnographie ou musée de société. À l'occasion de telles manifestations "grand public », la disipline ethnomusicologique elle-même peut aussi se revaloriser en remettant en question ses options épistémologiques pour devenir peut-être plus transparente et moins confidentielle, et par là même enrichir son champ d'investigation. 


\section{AUTEUR}

FRANÇOIS BOREL

François Borel est conservateur adjoint au Musée d'ethnographie de Neuchâtel, où il est responsable des collections d'instruments de musique, des collections d'Afrique sahélienne et des Archives sonores. Par ailleurs, il enseigne l'ethnomusicologie à l'Institut d'ethnologie de l'Université de Neuchâtel et au Lycée Denis-de-Rougemont. En tant que chercheur ethnomusicologue, il est spécialiste des musiques du Niger, en particulier celles des Touaregs. 